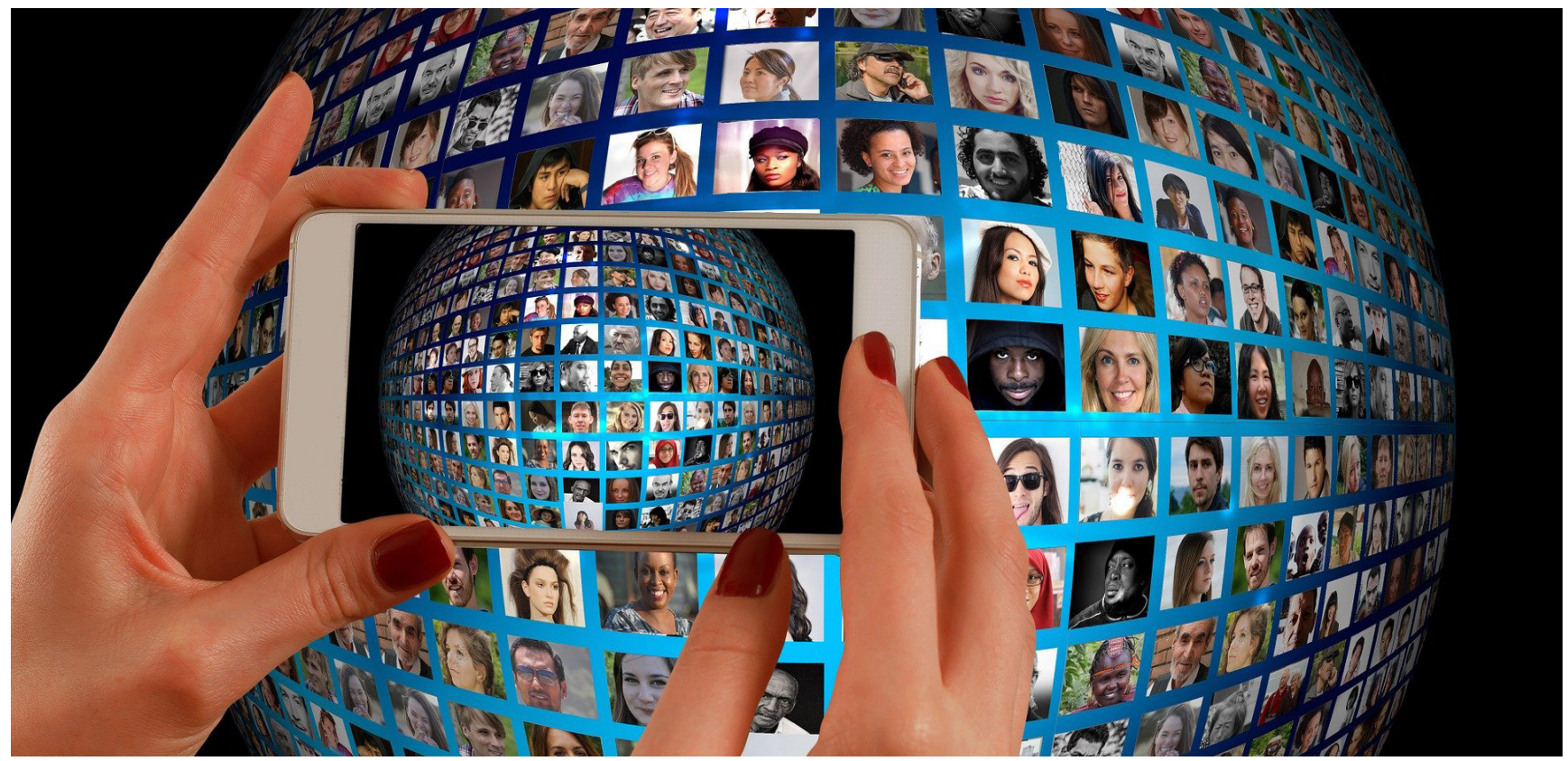

\title{
Gestión de intangibles en la industria informal de la moda en el subsector confecciones de Cúcuta
}

\section{Loss of management of intangibles in the informal fashion industry in Cucuta garment sub-sector}

Adriana Marcela Camacho-Castillo

Estudiante, Fundación de Estudios Superiores Comfanorte, Cúcuta, Colombia
Davinson Mauricio Torres-Mendoza

Estudiante, Fundación de Estudios Superiores Comfanorte, Cúcuta, Colombia

Ender José Barrientos-Monsalve

Doctor en Ciencias Gerenciales,

ej_barrientos@fesc.edu.co, Fundación de Estudios

Superiores Comfanorte, Cúcuta, Colombia 


\section{Resumen}

El presente trabajo tiene como objetivo analizar la relación de la informalidad sectorial de micro y pequeñas empresas de confecciones en la ciudad de Cúcuta con la capacidad de generar activos intangibles a través de la gestión de capital intelectual y su incidencia en la competitividad y en la creación de valor. Se realizó un estudio de tipo descriptivo a través de una revisión bibliográfica para sintetizar los conceptos clave que permiten la definición teórica de los activos intangibles y el capital intelectual desde diferentes enfoques disciplinares, se seleccionaron los factores determinantes para la gestión de activos intangibles y capital intelectual aplicables a la industria de la moda para desarrollar una entrevista a representantes sectoriales en la actividad gremial y de producción con respecto a sus percepciones formación y conocimientos académicos y/o empíricos en la generación y valoración de intangibles que les permitieran usarlos como herramientas para mejorar su competitividad, crear valor agregado y desarrollar ventajas competitivas a largo plazo. El resultado inicial denota parámetros básicos para la identificación de los factores de la gestión de activos intangibles en las micro y pequeñas empresas de las confecciones, su descripción cualitativa y definición conceptual para posteriormente ser analizados. Se concluye que hay una relación inversamente proporcional entre el nivel de conocimiento y uso del capital intelectual con la informalidad de las micro y pequeñas empresas de confecciones en Cúcuta, lo cual convierte en una necesidadpara la sostenibilidad y crecimiento del sector- la planeación de estrategias, sea desde las entidades, los gremios y/o la dirección de la organización, la identificación, comprensión, asimilación y aplicación sistemática en la actividad empresarial de la gestión de los activos intangibles y capital intelectual como herramienta de competitividad en la era de la información y el conocimiento.

Palabras clave: Activo intangible, capital intelectual, competitividad, industria de la moda, Informalidad.

\section{Abstract}

The objective of this paper is to analyze the relationship between the sectoral informality of micro and small apparel companies in the city of Cúcuta and their capacity to generate intangible assets through the management of intellectual capital and its impact on competitiveness and value creation. A descriptive study was conducted through a literature review to synthesize the key concepts that allow the theoretical definition of intangible assets and intellectual capital from different disciplinary approaches, the determining factors for the management of intangible assets and intellectual capital applicable to the fashion industry were selected to develop an interview to sectorial representatives in the guild and production activity regarding their perceptions, training and academic and/or empirical knowledge in the generation and valuation of intangibles that would allow them to use them as tools to improve their competitiveness, create added value and develop competitive advantages in the long term. The initial result denotes basic parameters for the identification of intangible asset management factors in micro and small apparel companies, their qualitative description and conceptual definition to be subsequently analyzed. It is concluded that there is an inversely proportional relationship between the level of knowledge and use of intellectual capital and the informality of micro and small apparel companies in Cúcuta, which makes it a necessity - for the sustainability and growth of the sector - the planning of strategies, whether from the entities, the guilds and/or the management of the organization, the identification, understanding, assimilation and systematic application in the business activity of the management of intangible assets and intellectual capital as a tool for competitiveness in the information and knowledge era.

Keywords: Informality, fashion industry, intellectual capital, intangible assets, competitivity 


\section{6}

\section{Introducción}

La informalidad es una manifestación de los procesos económicos de una zona geográfica y/o sector productivo definido, que en los últimos años se ha convertido en un indicador que permite analizar un aspecto social del desempeño económico, a nivel productivo, normativo y laboral con los respectivos efectos sobre la competitividad (Portes y Haller, 2014). La definición y caracterización de la informalidad en las unidades productivas es de reciente inclusión en la literatura económica a pesar de ser un fenómeno que se ha observado históricamente en diversos países desde la revolución industrial y su importancia actual radica en la descripción de las relaciones productivas con el objetivo de generar empleo, bienestar, conocimiento, rentabilidad y/o valor agregado a los productos o servicios en el entorno de lo que se ha denominado economía del conocimiento, la cual se basa en la creación o aumento del capital intangible en la conformación actual de las empresas como principal ventaja competitiva (Sandoval, 2014).

En este sentido, el capital intangible ha sido dado a describirse como los bienes inmateriales susceptibles de generar beneficios a futuro, comprende aquello que no tiene una existencia material y no se puede cuantificar pero se refleja en la contabilidad y tiene efectos en el funcionamiento de la empresa así como en su entorno competitivo y en el mercado; tales como la innovación, la creatividad, el liderazgo, las alianzas comerciales $\mathrm{y} / \mathrm{o}$ estratégicas, la organización o capital humano (Bello y otro, 2013). Los intangibles tienen relevancia especial dentro de determinadas industrias en razón a la naturaleza de las últimas, ya que determina el valor de las empresas por la capacidad de crear y desarrollar nuevos productos y procesos asociados a los mismos, es el caso de la industria de la moda que abarca subsectores donde la creatividad, el saber-hacer, la ID $+d$, la propiedad intelectual, el talento individual, la visión y la dirección, la marca y los secretos industriales entre otros atributos, son determinantes del éxito de una empresa (Dillon, 2012) al mismo tiempo, la moda integra diversas actividades económicas cuyas operaciones a nivel mundial, representan un mercado que equivale a la 7a economía en tamaño (formaría parte del G8) y dobla al del sector de electrónica y computación, una de las mayores en la creación de valor agregado y de intangibles y una de las más intensivas en mano de obra (Mamoq, 2018). Actualmente los centros de producción industrial de la moda se han trasladado a países con economías emergentes, dejando en los países industrializados la creación de intangibles que representan el mayor valor agregado en la industria, la cual está en crecimiento, mientras que el valor decrece en la producción de forma constante y es donde se busca reducir mayores costos.

En el caso de Colombia, los indicadores expresan comportamientos alentadores aunque las oportunidades de mejora siguen siendo un reto importante para la industria de la moda. Según la Superintendencia de Sociedades - Supersociedades (2017), el sector del Textil y Confección ha reflejado el dinamismo internacional, aportando en $8.8 \%$ del PIB del país en 2016 con un crecimiento del $3.0 \%$ con respecto al año anterior, sin embargo el DANE a través de la Encuesta Anual Manufacturera develó que durante ese mismo año la producción de confecciones decayó 5.9\% manteniendo esta tendencia negativa toda la cadena de valor en diferentes proporciones, también, la venta de confecciones registró una variación negativa de $-1.9 \%$. El no cumplimiento de las metas sectoriales trazadas por el Programa de Transformación Productiva - PTP del Ministerio de Comercio, Industria y Turismo en el Plan de Negocios 2009, motivó la actualización del documento en el año 2016 donde se realiza un diagnóstico de necesidades que se dividen en cuatro áreas: Capital humano, fortalecimiento industrial, marco normativo e infraestructura 
y sostenibilidad; todas con alto componente de creación de activos intangibles, capital intelectual y gestión de conocimiento: marca, Investigación y Desarrollo (I+D+i), transferencia tecnológica, formación del talento humano, creación de valor agregado, certificaciones y sistemas de gestión, Tecnologías de la Información (TIC).

La industria de la moda de Colombia, tiene como uno de sus núcleos a Cúcuta cuyo nutrido sector industrial de confecciones tiene sus orígenes en la fuerte vocación comercial derivada de la ventaja comparativa de ser una ciudad fronteriza con un mercado que durante muchos años mantuvo un alto nivel de vida; la constante y creciente demanda de artículos de moda como prendas de vestir, calzado y accesorios, hizo de Cúcuta un lugar propicio para la fabricación de estos productos debido a las ventajas por motivos de costos que representaba la tasa de cambio entre las monedas de Colombia y Venezuela. Según Suárez (2016) la actual situación política y económica de Venezuela ha deteriorado de forma ostensible la calidad de vida de las personasy, con el paso fronterizo restringido, los niveles de intercambio comercial decrecieron drásticamente, hechos que sumados a la inestabilidad económica y política que dificulta la realización de negocios. La necesidad de diversificar los mercados de las empresas, ha evidenciado el carácter marcadamente productor de la industria cucuteña que para mantenerse a flote debe iniciar su tránsito a la economía del conocimiento y generar las capacidades para la creación de intangibles (Ibídem).

Dentro de esta lógica, el siguiente paso para el sector industrial de las confecciones en Cúcuta, como resume Acosta, et al. (2016) es aumentar su competitividad a través de la innovación, valor agregado, diseño, calidad y marketing para lograr la fidelización de los clientes/ usuarios como estrategia para afrontar los grandes cambios que implica la globalización en la economía del conocimiento. Para establecer la relación entre la informalidad empresarial de la industria de la moda en Cúcuta con la capacidad de generar activos intangibles a través de la gestión del capital intelectual, primero se procederá a indagar la situación del conocimiento de activos intangibles y capital intelectual en las empresas de confecciones de Cúcuta, para posteriormente analizar los factores de la situación del conocimiento de Activos Intangibles y Capital Intelectual en las empresas y develar el uso y aplicación de los Activos Intangibles y Capital Intelectual.

La investigación propuesta plantea una aproximación al fenómeno de la informalidad y sus efectos sobre la capacidad de generar valor y activos intangibles con el fin de describir la disminución gradual de los precios de mercado de los artículos de moda fabricados por las empresas en lugares con vocación productiva, que actúan como proveedores y sin posicionamiento en el mercado, en contraposición con el aumento del valor agregado por las marcas líderes de la industria de la moda, las cuales demandan los artículos de los proveedores mencionados, al menor precio posible. Esta industria es dinamizadora de la economía en varios países debido a su requerimiento intensivo de mano de obra, sin embargo, la sostenibilidad económica y social no está garantizada por las condiciones de informalidad en que se desarrollan las labores en algunos países con legislación laboral que no contempla la generación de condiciones dignas para los trabajadores y es permisiva con prácticas que privilegian la necesidad de reducir costos sobre la remuneración justa.

En este orden de ideas, el análisis de las capacidades para la generación de capital intelectual y activos intangibles como estrategia para agregar valor, representaría una posibilidad de aumentar la competitividad de las empresas productoras de confecciones que conforman la cadena de valor de las marcas, a través de la especialización del 


\section{8}

capital humano, la aplicación de conocimiento y uso de tecnologías que configuren una propuesta de valor para el mercado, con lo cual estas empresas proveedoras pueden mejorar su posición negociadora ante una marca. Tal situación representaría un aumento en el precio y por lógica organizacional y de mercado, este iría en gran parte al personal que conforma o desarrolla el activo intangible.

A partir de lo mencionado, los resultados del presente trabajo pueden constituir el insumo en el desarrollo de un modelo de gestión de capital intangible para las empresas de confecciones proveedoras de marcas, que facilite la sistematización parcial de la creación de una propuesta de valor y/o la aplicación de una metodología en diversos entornos que permita mejorar las condiciones negociación y la remuneración así como las condiciones laborales, transformando en capital humano al personal que actualmente se desempeña como mano de obra en la industria de la moda.

\section{Marco Teórico}

La informalidad como fenómeno económico ha sido observada desde diversos enfoques y analizada a la luz de varias teorías. La definición que de la misma realizó por primera vez Keith Hart en 1973 en un informe elaborado para la OIT con ocasión de la investigación del mercado laboral urbano en África, es considerada el punto de partida de la caracterización de este fenómeno y de los parámetros para su medición y estudio. En la comparación de las estadísticas con respecto a la evolución de la estructura del mercado laboral a partir de datos comparativos de países altamente industrializados con otros menos industrializados, Sandoval (2014) concluye que la teoría económica clásica de origen marxista no explica el desempleo en las economías de mercado y su relación con la informalidad en el contexto contemporáneo.
En cuanto a la informalidad, tanto laboral como empresarial, son pobres los indicadores para Cúcuta y su área de influencia, por lo que establecer la relación entre este fenómeno y los bajos niveles de productividad y competitividad, es un tema que han abordado la Cámara de Comercio de Cúcuta y otros (2013) con miras a la fundamentación y generación de políticas dirigidas a la recuperación y fortalecimiento del tejido empresarial cuya estructura productiva, comercial y de servicios está conformada principalmente por pequeñas empresas, que usualmente ven limitado o estancado su crecimiento por factores competitivos, siendo propensas a operar bajo algún grado de informalidad en sus procesos para subsistir.

Las características de la zona objeto de esta investigación, han suscitado el análisis académico de la economía fronteriza, en áreas como la competitividad, productividad e informalidad al ser estos factores de la realidad empresarial y social que expresan los indicadores económicos. La generación de ventajas competitivas, estrategias de productividad e innovación con enfoque en el valor agregado, calidad y diseño para fidelización de clientes y usuarios, son planteadas como una necesidad para aumentar la competitividad y la productividad en las empresas del sistema moda de Cúcuta por Acosta y otros (2015), dado el bajo grado de compromiso e interés dentro de la industria hacia la ejecución de actividades en pro de la mejora en la eficiencia y eficacia de las empresas del sector, a través de la estandarización de procesos, y la ausencia de direccionamiento estratégico de estas unidades productivas, con base en la adecuada gestión del talento - capital humano en aras de aumentar la competitividad sectorial.

La industria de la moda es un sector en constante crecimiento y "se caracteriza por la innovación y uso intensivo de la creación y el conocimiento, lo que irremediablemente 
las hace propietarias y usuarias de un elevado volumen de intangibles y de indispensable capital humano", es la reflexión sobre el manejo de intangibles y capital social en la industria de la moda que realizan Bello y Echavarría (2013); los intangibles constituyen activos que deben ser evaluados y valorados correctamente para incluir en balances contables en la conformación del capital social de las empresas, tomando en cuenta que existan términos homogéneos para la valoración de los intangibles en la industria, ya que estos inciden en aspectos competitivos, legales y tributarios que representan una importante reserva para las marcas reconocidas pero son pasados por alto en las empresas pequeñas que son la mayoría en el tejido productivo.

\section{Activo intangible y capital intelectual}

El uso de estos dos conceptos ha sido objeto de debate para diversos autores y académicos y no se ha llegado a establecer una definición ampliamente aceptada, a pesar de que "la importancia y el conocimiento y del capital intelectual se conoce desde la antigüedad, donde civilizaciones como la griega o la egipcia presentan las primeras evidencias de codificación de conocimiento... como una forma de incrementar su poder regional" (Sánchez, et al. 2007), desde entonces y hasta la década del 90 del Siglo XX, cuando es notorio el interés por llegar a una conceptualización dentro de un marco soportado en las teorías existentes, la definición de tales conceptos ha representado un reto para los académicos (Ibídem). Según Simó y Sallán (2008), el capital intelectual se define "como conocimiento que crea valor, siendo por tanto un caso particular de activo intangible" y de acuerdo con Lev (2001) a pesar del amplio uso de los conceptos capital intelectual, activos intangibles y activos de conocimiento, su diferencia radica en el ámbito de la disciplina siendo preferido en la contabilidad el uso de activos intangibles, en economía el de activos de conocimiento y en administración el de capital intelectual.
Siguiendo esta línea, se establece que las definiciones que hablan de la representación de los activos en los balances financieros en la cuenta denominada fondo de comercio, tienen enfoque hacia las disciplinas contables y son insuficientes para abarcar la totalidad de elementos que se consideran intangibles y generan valor para la organización, aún cuando no sean registrados contablemente. Para ilustrar los diferentes enfoques de la definición de capital intelectual y activo intangible, se cita la metáfora de Edvinson y Malone (2009): "Una corporación es como un árbol. Hay una parte que es visible (las frutas) y una parte que está oculta (las raíces). Si solamente te preocupas por las frutas, el árbol puede morir. Para que el árbol crezca y continúe dando frutos, será necesario que las raíces estén sanas y nutridas. Esto es válido para las empresas: si sólo nos concentramos en los frutos (los resultados financieros) e ignoramos los valores escondidos, la compañía no subsistirá en el largo plazo".

En la actualidad, la mayoría de los autores acepta que los conceptos de capital intelectual y activos intangibles ha superado la limitación de su presencia en los estados financieros y basan sus aportes en la evolución de lo que han representado para las actividades económicas a lo largo de la historia y sus implicaciones en los tiempos actuales donde se está configurando un nuevo paradigma económico donde son cada vez más relevantes, tal como lo interpreta Peralta (2002) a partir del texto de Brooking (1997): "el capital intelectual no es nada nuevo, sino que ha estado presente desde el momento en que el primer vendedor estableció una buena relación con un cliente. Más tarde, se le llamó fondo de comercio. Lo que ha sucedido en el transcurso de las dos últimas décadas es una explosión en determinadas áreas técnicas claves, incluyendo los medios de comunicación y la tecnología de la información y las comunicaciones, que han 


\section{0}

proporcionado nuevas herramientas con las que se ha edificado una economía global".

Si bien se encuentran diferencias en los conceptos de activo intangible y capital intelectual, se evidencia que el último tiene relación con el conocimiento y su adecuada gestión para la crear valor a la empresa (Simó y Sallán, 2008).

\section{Informalidad}

De acuerdo con (De Soto, 1987), La informalidad es una categoría que nace de la observación empírica de un fenómeno, se trata de una zona de penumbra que comparte una extensa frontera con el mundo legal y "donde los individuos se refugian cuando los costos de cumplir las leyes exceden sus beneficios"

Cartaya (1987) habla de "elevar el nivel de vida de la población que vive y trabaja en la informalidad, potenciar los aspectos relacionados con la capitalización y productividad de los micronegocios y mejorar los sistemas de administración de los mismos". Un cuarto factor se fue agregando a medida que ganaban espacios las lecturas neoliberales del problema: la simplificación administrativa y el pago de impuestos. Es sabido que ese "confuso mundo del sector informal.

Estos autores determinan que la informalidad está integrada por un enorme universo de pequeños productores y trabajadores por cuenta propia que evaden el pago de impuestos y los compromisos de cotizar en las cuentas de los sistemas de seguridad social; un informe de la Organización Internacional del Trabajo (OIT), elaborado en 1972, ofreció la oportunidad de identificar y situar en el campo de la teoría y en el terreno de las políticas públicas a un sector social que escapaba a los paradigmas clásicos de empresarios y trabajadores; terratenientes o industriales; comerciantes y clases medias. Había indicios abrumadores de que una vasta capa de la población trabajadora de los países periféricos obtenía sus ingresos de actividades laborales que no estaban claramente definidas en los estudios y diagnósticos que servían de base para sustentar los programas de asistencia social y de combate a la pobreza. El tema había sido parcialmente tratado, por la sociología marxista, como un fenómeno de crecimiento desmesurado del trabajo no asalariado, y de formas atípicas de producción en las sociedades menos desarrolladas.

\section{Competitividad}

"El análisis de la competencia es un aspecto clave del necesario seguimiento de los mercados, éste aborda la evaluación de las fortalezas y debilidades de los competidores actuales y de los potenciales, una técnica muy utilizada, es la caracterización y clasificación de los competidores principales según los siguientes pasos: definir el negocio - el alcance y la naturaleza del negocio"

Por otra parte Mahmood (2000) sostiene que la ventaja competitiva es creada y apropiada por firmas individuales (características de bienes privados), "...claramente, uno no debe escoger entre uno de los dos paradigmas, pues no son ni mutuamente exclusivos ni explícitamente separables. Entonces, podemos sostener que es inapropiado presentar a la ventaja competitiva como una alternativa (sustituta) de la ventaja comparativa. Las dos teorías tienen que ser debidamente vistas como complementos más que como competidores en la formulación de políticas comerciales e industriales"

\section{Valor Agregado}

Como lo explican Castellano y Goizueta (2015), el Valor Agregado es la diferencia entre la producción y el consumo intermedio y representa la contribución de la mano de obray el capital al proceso productivo, este concepto está directamente vinculado con el producto bruto interno (P.I.B.), ya que este mide el total del valor agregado de todas las unidades 
institucionales de la economía; permitiendo realizar un análisis conceptual amplio sobre el tema explicando distintas iniciativas de valor agregado desde la perspectiva pública y privada incorporando el concepto "valor agregado institucional".

En cambio Porter y Kramer (2011) hablan sobre el "valor compartido" vinculando la competitividad de la empresa con la mejora de las condiciones sociales y económicas de la comunidad donde opera, y Champredonde y González Cosiorovski (2013) proponen un enfoque multidimensional a través del concepto "valor integral" de recursos territoriales, donde se traslada el foco de atención del producto hacia el conjunto de actores involucrados en un proceso de valorización, e implica considerar las actividades humanas en toda su complejidad.

Por otra parte, desde el punto de vista microeconómico, el valor agregado (V.A.) según el Diccionario de Oxford (s.f.) es "el monto por el cual el valor de un producto se incrementa en cada etapa de su producción, excluyendo los costos iniciales". La FAO lo define como "la diferencia entre lo que cuesta poner un producto de determinadas características en el mercado y lo que el cliente está dispuesto a pagar por él, o lo que éste percibe como valor", introduciendo en la definición el concepto de calidad.

El énfasis de estos autores es ampliar el significado del término "valor" y así permitir satisfacer la necesidad de proporcionar una gran pista a la hora de desarrollar nuestros productos, es decir permite conocer cuáles son los atributos que valoran nuestros clientes, cuáles son sus necesidades y qué problema quieren resolver, para seguir avanzando en el mercado.

\section{Materiales y métodos}

El presente estudio es de tipo cualitativo y de alcance descriptivo. Se obtiene la información inicial de fuentes documentales por medio de revisión bibliográfica para sintetizar los conceptos clave que permiten la definición teórica de los activos intangibles y el capital intelectual desde diferentes enfoques disciplinares.

La recolección de los datos se realiza por medio de entrevistas a micro y pequeños empresarios de las confecciones con participación en una entidad gremial específica y al representante de la misma. El análisis de la información es plasmado a través de la tabulación de las respuestas con la respectiva conclusión donde se establece la relación entre los conceptos teóricos y el fenómeno investigado.

\section{Resultados y análisis}

Las entrevistas fueron realizadas a propietarios y/o administradores de micro y pequeñas empresas de confecciones agremiadas en una entidad al representante de la misma por ser partícipes y sujetos de los fenómenos objeto de estudio conceptualizados en el marco teórico. El grupo seleccionado ha demostrado interés en aumentar su productividad, competitividad e implementar estrategias para tales fines, solicitando acompañamiento de instituciones educativas y entidades sectoriales en proyectos colectivos e individuales. 
Tabla 1. Análisis de respuestas a entrevista.

\begin{tabular}{|c|c|c|c|}
\hline & & ENTREVISTA & \\
\hline PREGUNTA & INFORMANTES & RESPUESTA & ANALISIS \\
\hline \multirow[b]{2}{*}{$\begin{array}{l}\text { 1. ¿Qué ha } \\
\text { escuchado sobre } \\
\text { el concepto de } \\
\text { capital/actrivo } \\
\text { intangible? }\end{array}$} & Informante 1 & No conoce el concepto & La identificación del concepto \\
\hline & Informante 4 & $\begin{array}{l}\text { Ha escuchado sobre } \\
\text { capital intangible en } \\
\text { capacitaciones sobre } \\
\text { otros temas } \\
\text { empresariales y sabe } \\
\text { que es importante para } \\
\text { lograr determinados } \\
\text { resultados en el largo } \\
\text { plazo leido } \\
\text { Ha sobre } \\
\text { superficialmente lo } \\
\text { el concepto y los } \\
\text { relaciona con has } \\
\text { avances tecnologicos } \\
\text { De diversas fuentes ha } \\
\text { obtenido conceptos } \\
\text { generales y sabe que es } \\
\text { un aspecto a desarrollar } \\
\text { para diferenciarse }\end{array}$ & $\begin{array}{l}\text { existe pero su definición es } \\
\text { nula o ambigua e incierta en } \\
\text { términos generales para los } \\
\text { entrevistados. Esto refleja que } \\
\text { el interés académico que en } \\
\text { décadas recientes ha } \\
\text { despertado el estudio y } \\
\text { descripción del capital } \\
\text { intangible y capital } \\
\text { intelectual, se manifiesta en } \\
\text { su uso o adopción para la } \\
\text { justificación de proyectos } \\
\text { públicos y privados } \\
\text { orientados hacia el } \\
\text { fortalecimicnto del sector } \\
\text { productivo donde han } \\
\text { participado estos empresarios }\end{array}$ \\
\hline \multirow{8}{*}{$\begin{array}{l}\text { 2. ¿Qué entiende } \\
\text { usted por } \\
\text { capital/activo }\end{array}$} & Infor & No & \multirow{10}{*}{$\begin{array}{l}\text { La comprensión de los } \\
\text { factores asociados a los } \\
\text { activos intangibles, refleja el } \\
\text { uso de ideas muy amplias y de } \\
\text { origen intuitivo por los } \\
\text { términos que componen el } \\
\text { concepto. No se evidencia } \\
\text { reconocimiento con algún } \\
\text { grado de detalle de los } \\
\text { elementos básicos que definen } \\
\text { el activo intangible y el } \\
\text { capital intelecual } \\
\text { En la asimilación del proceso } \\
\text { de gestón de capital intangible } \\
\text { se observa una aproximación } \\
\text { similar al de la identificación } \\
\text { del concepto (pregunta 1) } \\
\text { donde por asociación de los } \\
\text { términos y su relación con las } \\
\text { iniciativas y proyectos de }\end{array}$} \\
\hline & Informante 6 & No conoce el concepto & \\
\hline & Informante 1 & $\begin{array}{l}\text { Conocimiento, valor } \\
\text { agregado }\end{array}$ & \\
\hline & Informante 2 & Innovacion & \\
\hline & Informante 3 & Recursos nuevos & \\
\hline & $\begin{array}{l}\text { Informante } 4 \\
\text { Informante } 5\end{array}$ & $\begin{array}{l}\text { Innovacion } \\
\text { Valor agregado a cada } \\
\text { proyecto }\end{array}$ & \\
\hline & Informante 6 & $\begin{array}{l}\text { Es un valor agregado el } \\
\text { cual no se puede tocar }\end{array}$ & \\
\hline & & per & \\
\hline $\begin{array}{l}\text { 3. ¿Por qué } \\
\text { considera } \\
\text { importante para } \\
\text { su empresa }\end{array}$ & Informante 1 & $\begin{array}{l}\text { Para ser mejores en las } \\
\text { actividades de negocio y } \\
\text { mejorar los ingresos por } \\
\text { ventas de sus productos }\end{array}$ & \\
\hline $\begin{array}{l}\text { perteneciente al } \\
\text { sector de la moda, } \\
\text { la gestión de } \\
\text { capital }\end{array}$ & Informante 2 & $\begin{array}{l}\text { Porque permite el } \\
\text { desarrollo de atributos } \\
\text { para diferenciarse de la } \\
\text { competencia }\end{array}$ & \\
\hline
\end{tabular}




\begin{tabular}{|c|c|c|c|}
\hline \multirow[t]{5}{*}{ intangible? } & Informante 3 & $\begin{array}{l}\text { Porque permite la } \\
\text { generacion de nuevas } \\
\text { fuentes de ingresos para } \\
\text { el crecimiento de la } \\
\text { empresa }\end{array}$ & $\begin{array}{l}\text { fomento empresarial, hay una } \\
\text { predisposición positiva hacia } \\
\text { la gestión de conocimiento } \\
\text { pero no una nocion clara } \\
\text { sobre los efectos concretos }\end{array}$ \\
\hline & Informante 4 & $\begin{array}{l}\text { Porque es algo } \\
\text { novedoso y por lo tanto } \\
\text { representa una ventaja }\end{array}$ & $\begin{array}{l}\text { que tendria sobre la } \\
\text { organización o empresa }\end{array}$ \\
\hline & Informante 5 & $\begin{array}{l}\text { Porque implica un } \\
\text { trabajo a largo plazo y } \\
\text { aumento de ingresos en } \\
\text { el tiempo } \\
\text { Porque tiene efecto en } \\
\text { los estados financieros c } \\
\text { indicadores de gestión }\end{array}$ & \\
\hline & Informante 1 & $\begin{array}{l}\text { Registro de marca, } \\
\text { contratos de cesión de } \\
\text { marca }\end{array}$ & $\begin{array}{l}\text { Como complemento a la } \\
\text { pregunta } 2 \text { para indagar sobre } \\
\text { la asimilación de algin }\end{array}$ \\
\hline & Informante 2 & $\begin{array}{l}\text { No sabe cómo } \\
\text { registrar/medir la } \\
\text { gestion de su capital } \\
\text { intangible } \\
\text { Registro de marca, } \\
\text { Franquicias, Inventos } \\
\text { industriales, gestion de } \\
\text { conocimiento, contratos } \\
\text { de cesión de imagen }\end{array}$ & $\begin{array}{l}\text { concepto sobre activos } \\
\text { intangibles, se develo que } \\
\text { ninguna de las empresas tiene } \\
\text { claridad para la evaluación, } \\
\text { medición y valoración de su } \\
\text { capital intelectual aún cuando } \\
\text { algunas cuentan con } \\
\text { elementos definidos como } \\
\text { intangibles y por lo tanto no }\end{array}$ \\
\hline \multirow{3}{*}{$\begin{array}{l}\text { ¿Cómo realiza en } \\
\text { su empresa alguin } \\
\text { tipo de gestión } \\
\text { verificable de } \\
\text { capitales/activos } \\
\text { intangibles? }\end{array}$} & Informante 4 & $\begin{array}{l}\text { No sabe cómo } \\
\text { registrar medir } \\
\text { gestión de su capital } \\
\text { intangible }\end{array}$ & $\begin{array}{l}\text { son conscientes de si su } \\
\text { empresa posec cste tipo de } \\
\text { activos o no. Tambien se } \\
\text { interpreta como la ausencia de }\end{array}$ \\
\hline & Informante 5 & $\begin{array}{l}\text { No sabe cómo } \\
\text { registrar/medir la } \\
\text { gestión de su capital } \\
\text { intangible }\end{array}$ & $\begin{array}{l}\text { la aplicación de procesos de } \\
\text { gestión de capital intangible y } \\
\text { por lo tanto una falencia } \\
\text { (desventaja) dentro del }\end{array}$ \\
\hline & Informante 6 & $\begin{array}{l}\text { No sabe como } \\
\text { registrar/medir la } \\
\text { gestión de su capital } \\
\text { intangible }\end{array}$ & $\begin{array}{l}\text { entorno competitivo de estas } \\
\text { empresas. }\end{array}$ \\
\hline \multirow{2}{*}{$\begin{array}{l}\text { 4. ¿Cuales casos } \\
\text { de excito en la } \\
\text { gestión de capital } \\
\text { intangible que } \\
\text { conozca podria } \\
\text { implementar en }\end{array}$} & Informante 1 & $\begin{array}{l}\text { No tiene identificados } \\
\text { casos de éxito aplicables } \\
\text { a su empresa }\end{array}$ & $\begin{array}{l}\text { El tener referencias sobre } \\
\text { casos extrapolables se } \\
\text { interpreta como un indicio de }\end{array}$ \\
\hline & Informante 2 & $\begin{array}{l}\text { No tiene identificados } \\
\text { casos de éxito aplicables } \\
\text { a su empresa. }\end{array}$ & $\begin{array}{l}\text { intención de aplicación o } \\
\text { implementación de la gestión } \\
\text { de intangibles que podria }\end{array}$ \\
\hline
\end{tabular}


64

\begin{tabular}{|c|c|c|c|}
\hline \multirow[t]{6}{*}{ su empresa? } & Informante 3 & $\begin{array}{l}\text { No tiene identificados } \\
\text { casos de exito aplicables } \\
\text { a su empresa }\end{array}$ & \multirow{4}{*}{$\begin{array}{l}\text { materializarse en un marco de } \\
\text { tiempo cercano. Se observa } \\
\text { que no hay referentes a seguir } \\
\text { y que si bien la intención } \\
\text { puede estar presente, aun no } \\
\text { tiene forma definida que } \\
\text { permita pensar que sera una } \\
\text { realidad en el corto o mediano } \\
\text { plazo. }\end{array}$} \\
\hline & Informante 4 & $\begin{array}{l}\text { No tiene identificados } \\
\text { casos de exito aplicables } \\
\text { a su empresa. }\end{array}$ & \\
\hline & Informante 5 & $\begin{array}{l}\text { No tiene identificados } \\
\text { casos de éxito aplicables } \\
\text { a su empresa }\end{array}$ & \\
\hline & Informante 6 & $\begin{array}{l}\text { No tiene identificados } \\
\text { casos de éxito aplicables } \\
\text { a su empresa }\end{array}$ & \\
\hline & Informante 1 & $\begin{array}{l}\text { Capacitación del capital } \\
\text { humano }\end{array}$ & \multirow{6}{*}{$\begin{array}{l}\text { Esta fue una respuesta guiada } \\
\text { por medio de opciones } \\
\text { predefinidas para indagar } \\
\text { sobre la profundidad de la } \\
\text { implementación o aplicación } \\
\text { de procesos de gestión de } \\
\text { capital intelectual y se } \\
\text { observa que hay falta de } \\
\text { preparación a nivel de } \\
\text { formación y capacitación del } \\
\text { talento humano (incluidos los } \\
\text { propios entrevistados) en el } \\
\text { uso de estos conceptos para el } \\
\text { beneficio de la empresa. }\end{array}$} \\
\hline & Informante 2 & $\begin{array}{l}\text { Capacitación del capital } \\
\text { humano }\end{array}$ & \\
\hline \multirow{4}{*}{$\begin{array}{l}\text { 5. ¿Qué método o } \\
\text { herramienta } \\
\text { emplearia usted } \\
\text { para la gestión de } \\
\text { intangibles en su } \\
\text { empresa? }\end{array}$} & Informante 3 & $\begin{array}{l}\text { Gestion } \\
\text { conocimiento }\end{array}$ & \\
\hline & Informante 4 & $\begin{array}{l}\text { Capacitación del capital } \\
\text { humano }\end{array}$ & \\
\hline & Informante 5 & $\begin{array}{l}\text { Capacitación del capital } \\
\text { humano }\end{array}$ & \\
\hline & Informante 6 & $\begin{array}{l}\text { Capacitación del capital } \\
\text { humano }\end{array}$ & \\
\hline
\end{tabular}

La realización de las entrevistas permitió experimentar de primera mano la confusión que genera la conceptualización de los activos intangibles y capital intelectual entre los empresarios, la situación tiene sentido si se analiza en paralelo con el proceso de definir el marco teórico, que no ha sido un recorrido rápido y en línea recta para la academia y se encuentra en construcción, con lo que se puede inferir que el sector productivo es sujeto de unas fuerzas que aún no están plenamente estudiadas y sin embargo ejercen una influencia notable en las actividades de producción, de comercialización y de direccionamiento estratégico a los nuevos escenarios donde se gestan los negocios de la industria, tales como la creación de valor, la transformación o adaptación tecnológica en relación con el mercadeo y el servicio al cliente que exige formas novedosas de concebir la actividad de la empresa a través del desarrollo de un modelo de negocio con capacidad de adaptación a los requerimientos del mercado, en un entorno de alta competencia a nivel global.

\section{Conclusiones}

Las empresas ya no son vistas o aprehendidas como un conjunto de infraestructura física y de recursos materiales que reposan en oficinas, bodegas o cuentas bancarias para conocer la realidad tras el comportamiento de la industria de la moda, es necesario tener en cuenta la gestión de los activos intangibles y de capital intelectual. La relevancia que han tomado estos factores y el volumen de literatura producido en los últimos años explicando sus efectos y su manejo para el logro de determinados objetivos, hace que, incluso sin ser conscientes de ello, gran parte de la industria ya esté inmersa en algún tipo de acercamiento con los activos intangibles y que construya conocimiento y capital intelectual que aún no hace parte de sus estados financieros. 
En cuanto a las micro y pequeñas empresas de confecciones como agentes de una industria con un alto nivel de crecimiento y creadora intensiva de capitales intangibles, su situación de expectativa ante lo que representa este nuevo tipo degestiónyel proceso deadaptación a la nueva economía del conocimiento los ha convertido en espectadores de los altos beneficios que representa para quienes se han iniciado en la consolidación de sus activos intangibles y capital intelectual desde tiempo atrás y lo perciben en el crecimiento espectacular de marcas de transnacionales que han deslocalizado su producción y gracias a internet han incursionado en mercados con cada vez más rapidez, convirtiendo a estas micro y pequeñas empresas en proveedoras dentro de los modelos de negocio desarrollados por las mencionadas marcas.

La situación de Cúcuta en particular involucra factores derivados de la transición económica de la industria de la moda ante los efectos generados por los cambios en la dinámica fronteriza; donde los históricos niveles de informalidad empresarial y la competitividad -dada principalmente por el tipo de cambio de la moneda de su mercado histórico- pueden ser mitigados a través de la gestión de activos intangibles y capital intelectual configurando un escenario para validar la relación entre los intangibles y el aumento de la competitividad planteada en el presente estudio, según Stewart (1998) "la gestión del conocimiento es el conjunto de procesos que hacen que el Capital Intelectual de la empresa crezca" por lo tanto es una herramienta necesaria para el adecuado aprovechamiento de la actividad que ya realizan las empresas de confecciones en su sector, generando y consolidando los activos intangibles de terceros, y que haría sostenible cualquier estrategia regional $\mathrm{o}$ particular tendiente al fortalecimiento de la industria en términos de excelencia, innovación y valor agregado.
65

Es importante tener en cuenta que debido a la naturaleza misma del objeto de estudio y su carácter elusivo en el paradigma de la actividad de la industria de la moda en Cúcuta que, según el análisis de las entrevistas, aún no está siendo identificado, asimilado y aplicado para su aprovechamiento y aumento, un punto de partida válido es la caracterización de la cadena de valor de las confecciones para conocer la estructura del tejido empresarial y realizar una intervención precisa y alineada con las capacidades ya generadas por las empresas para satisfacer los requerimientos actuales y futuros de los mercados objetivo.

Por último, enfatizar que el presente estudio busca llevar a un análisis para describir unos indicadores que son valorados dentro de la economía del conocimiento y por lo tanto el objetivo amplio es contribuir a la incorporación del sector de las confecciones de Cúcuta en el mercado global creando valor y cambiando el horizonte de visión hacia una frontera con el mundo.

\section{Referencias}

Acosta, L., Jaimes, D., Vargas, B., \& Velásquez, M. (2015). Comparativa de competitividad del sector de la moda entre Cúcuta y el resto del país. Revista Convicciones, 2(4), pp. 47 - 56. Descargado http://www.fesc.edu.co/Revistas/ OJS/index.php/convicciones/issue/view/15

Albouy, D., \& Adesida, O. (2018). Sustainable fashion blueprint 2018. Obtenido de https:// www.mamoq.com/journal/sustainable-fashionblueprint-2018/

Bello, S., \& Echavarría, P. (2013). Los intangibles y el capital social en las empresas de la moda. En Memorias XII Congreso Argentino de Derecho Societario y VIII Congreso Iberoamericano de Derecho Empresario y de la Empresa. Buenos Aires. Acceso: http://www.susybelloknoll.com/ bienes-intangibles-moda/\#_ftnref29 


\section{6}

Brooking, A. (2004). El capital intelectual. Barcelona: Paidós.

Cámara de Comercio de Cúcuta. (2013). Estudio sobre la informalidad empresarial en el municipio de Cúcuta y su área de influencia. Cúcuta: Universidad de Santander - UDES. Obtenido de http://www.datacucuta.com/index. php/informalidad/535-estudio-2013

Cartaya, V. (1987). El confuso mundo del sector informal. En Nueva Sociedad No 90, Caracas, 7-8. Obtenido de https://nuso.org/articulo/elconfuso-mundo-del-sector-informal/

Champredonde, M. y Gonzalez Cosiorovski, J. (2013). ¿Agregado de Valor o Valorización integral? Reflexiones apartirdeDenominaciones de Origen en América Latina. VIII Jornadas Interdisciplinarias de Estudios Agrarios y Agroindustriales. Buenos Aires. Acceso: http:// www.redalyc.org/pdf/4695/469546924008.pdf

De Soto, H. (1987). El otro sendero: la revolución informal. México: Editorial Diana.

Dillon, S. (2012). Principios de gestión en empresas de moda. Barcelona: Editorial Gustavo Gili.

Edvinsson, L., \& Malone, M. (2003). El capital intelectual. Barcelona: Gestión 2000.

Lev, B. (2001). Intangibles: Management, Measurement And Reporting, Bookings Institution Press.

Mahmood, A. \& Fidelis Ezeala, H. (2000). Comparative versus competitive advantage, and competitiveness in developing countries. En: Socioeconomic Development in the 21st Century. Calcuta: International Institute for Development Studies, pp.241-256.

Peralta Alemán, G. (2002). De la filosofía de la calidad al sistema de mejora contínua. México:
Panorama.

Portes, A. \& Haller, W. (2004). La Economía Informal. Santiago de Chile: Cepal. Obtenido de https://repositorio.cepal.org/handle/11362/6091

Salvador, G. (2016). Agregado de valor: Compartiendo conceptos. Acceso https://inta. gob.ar/sites/default/files/agregado_de_valor. compartiendo_conceptos.pdf

Sánchez, A., Melián, A. y Hormiga E. (2007). El concepto de capital intelectual y sus dimensiones. Investigaciones Europeas De Dirección Y Economía De Empresa, 13(2), 97-111. Obtenido de http://www.redalyc.org/ articulo.oa? $\mathrm{id}=274120280005$

Sandoval, G. (2014). La informalidad laboral: causas generales. Equidad \& Desarrollo (22), pp. 9-45. Descargado de https://dialnet.unirioja. es/descarga/articulo/5166528.pdf

Simó, P., \& Sallán, J. (2008). Capital intangible y capital intelectual: Revisión, definiciones y líneas de investigación. Estudios De Economía Aplicada, 26(2), pp. 65 - 78. Obtenido de: http:// www.redalyc.org/html/301/30113187004/

STEWART, T. (1998). La nueva riqueza de las organizaciones: el capital intelectual. Ediciones Granica.

Suarez, E. (2016). Diagnóstico de la situación del desarrollo económico de Cúcuta durante las dos últimas administraciones, como base para la creación de una Zona de Régimen Aduanero especial en la ciudad. Bogotá: Universidad Colegio Mayor de Nuestra Señora del Rosario. Obtenido de: http://repository.urosario.edu.co/ handle/10336/13085

Superintendencia de Sociedades. (2017). Desempeño del sector textil - confección. Bogotá: Delegatura de Asuntos Económicos y Contables. Obtenido de https://incp.org.co/Site/ 
publicaciones/info/archivos/Textiles.pdf 